\title{
Revising Reverse-Phase Chromatographic Behavior for Efficient Differentiation of Both Positional and Geometrical Isomers of Dicaffeoylquinic Acids
}

\author{
Keabetswe Masike, ${ }^{1}$ Ian Dubery, ${ }^{1}$ Paul Steenkamp $\mathbb{D D}^{1,2}$ Elize Smit, ${ }^{3}$ and Edwin Madala ${ }^{1}{ }^{1}$ \\ ${ }^{1}$ Department of Biochemistry, University of Johannesburg, P.O. Box 524, Auckland Park 2006, South Africa \\ ${ }^{2}$ CSIR Biosciences, Natural Products and Agro-Processing Group, Pretoria 0001, South Africa \\ ${ }^{3}$ Department of Chemistry, University of Johannesburg, P.O. Box 524, Auckland Park 2006, South Africa
}

Correspondence should be addressed to Edwin Madala; emadala@uj.ac.za

Received 31 July 2017; Accepted 31 October 2017; Published 11 January 2018

Academic Editor: Josep Esteve-Romero

Copyright (c) 2018 Keabetswe Masike et al. This is an open access article distributed under the Creative Commons Attribution License, which permits unrestricted use, distribution, and reproduction in any medium, provided the original work is properly cited.

\begin{abstract}
Dicaffeoylquinic acids (diCQAs) are plant metabolites and undergo trans-cis-isomerization when exposed to UV irradiation. As such, diCQAs exist in both trans- and cis-configurations and amplify the already complex plant metabolome. However, analytical differentiation of these geometrical isomers using mass spectrometry (MS) approaches has proven to be extremely challenging. Exploring the chromatographic space to develop possible conditions that would aid in differentially separating and determining the elution order of these isomers is therefore imperative. In this study, simple chromatographic parameters, such as column chemistry (phenyl versus alkyl), mobile phase composition (methanol or acetonitrile), and column temperature, were investigated to aid in the separation of diCQA geometrical isomers. The high-performance liquid chromatography photodiode array (HPLCPDA) chromatograms revealed four isomers post UV irradiation of diCQA authentic standards. The elution profile/order was seen to vary on different reverse-phase column chemistries (phenyl versus alkyl) using different mobile phase composition. Here, the elution profile/order on the phenyl-derived column matrices (with methanol as the mobile phase composition) was observed to be relatively reproducible as compared to the alkyl $\left(\mathrm{C}_{18}\right)$ columns. Chromatographic resolution of diCQA geometrical isomers can be enhanced with an increase in column temperature. Lastly, the study highlights that chromatographic elution order/profile cannot be relied upon to fathom the complexity of isomeric plant metabolites.
\end{abstract}

\section{Introduction}

Dicaffeoylquinic acids (diCQAs) are plant secondary metabolites that are part of the family of bioactive metabolites called chlorogenic acids. Dicaffeoylquinic acids (diCQAs) are formed from an esterification reaction between quinic acid and 2 units of the hydroxycinnamic acid (HCA) derivative, caffeic acid $[1,2]$. It is reported that HCA derivatives such as caffeic acid are initially synthesized in the transconfiguration through the phenylpropanoid pathway [3]. However, due to the 1,2-disubstituted alkenic molecular structure (carbon-carbon double bond), these molecules absorb light (UV light) at a specific wavelength and readily convert to the cis-geometry [3-7].
Previously, cis-isomers of phenylpropanoid derivatives were understood to exist in minor quantities and, as such, were thought to be biologically insignificant [3]. However, due to environmental changes, these isomers may be prominent in plant organs (i.e., leaves and fruits) that are exposed to the sun's UV rays [6] and, in some cases, exist in equal proportions to the trans-isomer. These new photochemically produced products (cis-isomers) amplify the already complex plant metabolome. Thus, studies devoted to exploring the biological significance of $c i s$-isomers relative to their trans-counterparts have become necessary [8-13]. For instance, it has been shown that the phenylpropanoid pathway metabolite, trans-cinnamic acid, has less potent antituberculosis activity than its cis-counterpart, which is 
<smiles>O=C(/C=C/c1ccc(O)c(O)c1)O[C@H]1C(O)C[C@@](O)(C(=O)O)C[C@H]1OC(=O)/C=C/c1ccc(O)c(O)c1</smiles><smiles>O=C(/C=C/c1ccc(O)c(O)c1)O[C@H]1C[C@@](O)(C(=O)O)C[C@@H](OC(=O)/C=C/c2ccc(O)c(O)c2)[C@@H]1OC(=O)/C=C/c1ccc(O)c(/C=C/C(=O)O[C@@H]2C[C@](O)(C(=O)O)C[C@H](O)[C@H]2O)c1</smiles>

SCHEme 1: Chemical structures of the trans-form positional isomers of dicaffeoylquinic acids (diCQAs), namely, 1,3-diCQA, 1,5-diCQA, 3,4-diCQA, 3,5-diCQA, and 4,5-diCQA.

approximately 120 -fold more than the trans-form [8]. In addition, in vacuo studies have demonstrated that HCA derivatives, such as dicaffeoyltartaric acid (chicoric acid) and diCQA, possess anti-human immunodeficiency virus (HIV) type 1 DNA integrase activity, with the biological activity attributed to their cis-isomers [10-12]. However, currently, only a few in vitro and in vivo studies, that show the biological activities of cis-isomers of HCA derivatives, exist. This is possibly due to the lack of knowledge about the existence of $c i s$-isomers of HCA derivatives or the lack of cisform commercial standards [3].

Authentic standards of most of the HCA derivatives (trans-isomers), such as diCQAs, are commercially available, and these standards can be used to produce their ciscounterparts through the process of photoisomerization [4, $6,12,14,15]$. Identification of these related geometrical compounds using analytical techniques such as liquid chromatography linked to mass spectrometry (LC-MS) has proven impossible as they produce similar/identical MS fragmentation patterns [4]. As such, these analytical challenges have driven efforts in exploring the chromatographic space to suggest possible conditions that would aid in differentially separating and identifying these isomers $[4,12]$. In this endeavor, Clifford et al. UV irradiated five different authentic standards of diCQA positional isomers, namely, 1,3-diCQA, 1,5-diCQA, 3,4-diCQA, 3,5-diCQA, and 4,5diCQA, and analyzed the samples on a phenyl-hexyl column using aqueous acetonitrile as part of the mobile phase composition [4]. From the study, Clifford et al. anticipated three possible cis-isomers for all five of the diCQA positional isomers. The number of cis-isomers can be attributed to the stereochemistry of the quinic acid unit (positions 1, 3, 4, and 5) to which the caffeic acid units are esterified $[4,14]$ (Scheme 1). As such, two asymmetrical mono-cis-isomers (resulting from cis-isomerization on the respective caffeoyl arms on the quinic acid unit) and one di-cis-isomer (resulting from the cis-geometry on both caffeoyl arms on the quinic acid unit) were anticipated for each diCQA positional isomer. However, in the study by Clifford et al., only two (instead of three) cis-isomers were observed for 1,3diCQA, 3,4-diCQA, and 3,5-diCQA positional isomers [4].

Fundamentally, column chemistry $[12,16,17]$, mobile phase composition $[12,16]$, and column temperature $[18,19]$ are the essential factors in defining chromatographic separation during LC analyses. For instance, under reversephase conditions, a phenyl-derived column matrix may produce a different elution profile (i.e., number of isomers separated) compared to an alkyl-derived column matrix, due to the interactions involved in the retention mechanism of the respective columns $[12,16]$.

The aims of the current study were to reproduce and expand on the results observed by Clifford et al. [4]. In our study, the abovementioned positional isomers of diCQAs (1,3-diCQA, 1,5-diCQA, 3,4-diCQA, 3,5-diCQA, 
and 4,5-diCQA) were UV irradiated, and the resulting samples were analyzed on nine different column chemistries, five phenyl-derived columns and four alkyl $\left(\mathrm{C}_{18}\right)$ columns. The effect of chromatographic parameters (column choice, mobile phase composition, and column temperature) on the chromatographic separation of the UV-generated diCQA geometrical isomers was evaluated. Insight on the chromatographic elution order of these metabolites (both transand cis-isomers) will contribute to ongoing efforts in designing analytical methods for differential identification of isomers contributing to plant sample dimensionality. Such chromatographic separation efforts will further allow collection (LC fractionation) of these peaks (metabolites) to study their respective bioactivity differences.

\section{Materials and Methods}

2.1. Materials. Authentic standards (with the purity of above 99.6\%) of trans-dicaffeoylquinic acid (1,3-diCQA, 1,5-diCQA, 3,4-diCQA, 3,5-diCQA, and 4,5-diCQA) were purchased from Phytolab (Vestenbergsgreuth, Germany). Mass spectrometry grade $(99.9 \%)$ methanol and acetonitrile were obtained from Romil (Cambridge, UK). Mass spectrometry grade formic acid was purchased from Sigma-Aldrich (St. Louis, MO, USA). A UV light box Model CM-10 was purchased from Spectroline (Westbury, NY, USA). Pinnacle, Raptor, Viva, and Ultra columns were purchased from Restek (Bellefonte, PA, USA) and Kinetex columns were purchased from Phenomenex (Torrance, CA, USA). Chromatographic separation was achieved using nine analytical columns: Pinnacle bi-phenyl and $\mathrm{C}_{18}(2.1 \times 100 \mathrm{~mm}, 3 \mu \mathrm{m})$, Raptor biphenyl and $\mathrm{C}_{18}(2.1 \times 100 \mathrm{~mm}, 2.7 \mu \mathrm{m})$, Viva bi-phenyl $(2.1 \times 100 \mathrm{~mm}, 5 \mu \mathrm{m})$, Viva $\mathrm{C}_{18}(2.1 \times 100 \mathrm{~mm}, 3 \mu \mathrm{m})$, Ultra $\mathrm{C}_{18}(2.1 \times 100 \mathrm{~mm}, 3 \mu \mathrm{m})$, and Kinetex bi-phenyl and phenylhexyl $(4.6 \times 100 \mathrm{~mm}, 5 \mu \mathrm{m})$.

\subsection{Methods}

2.2.1. UV Irradiation. A $1 \mathrm{mg} / \mathrm{mL}$ solution of each transdiCQA positional isomer was prepared with $100 \%$ methanol. Irradiation of samples was conducted following the procedure described elsewhere [15]. Briefly, the solution (for each positional isomer) was placed in a Spectroline UV lamp operating at $254 \mathrm{~nm}$ with an intensity of $390 \mu \mathrm{W} / \mathrm{cm}^{2}$. The lamp was not covered with any notch filter. Irradiation was conducted for four hours $(4 \mathrm{~h})$, and aliquots $(100 \mu \mathrm{L})$ were taken at $0 \mathrm{~h}$ (before irradiation) and at $4 \mathrm{~h}$ post irradiation. The aliquots were diluted $10 \times$ with $100 \%$ methanol. All the samples were placed in amber vials and subjected to HPLCPDA analyses.

2.2.2. HPLC-PDA Parameters. The HPLC system used was a Shimadzu SCL-10A VP (Kyoto, Japan), equipped with a PDA controlled by Shimadzu VP software v. 5.31. Column oven temperature was set at $30^{\circ} \mathrm{C}$ and $50^{\circ} \mathrm{C}$. The injection volume was $3 \mu \mathrm{L}$. A binary solvent mixture was used, consisting of Milli-Q water (eluent A) containing $0.1 \%$ formic acid and methanol or acetonitrile (eluent B) containing $0.1 \%$ formic acid. The initial conditions were $10 \% \mathrm{~B}$ at a flow rate of $0.2 \mathrm{~mL} / \mathrm{min}$ and were maintained for $1 \mathrm{~min}$, followed by an increase to $40 \% \mathrm{~B}$ at $15 \mathrm{~min}$; the conditions were maintained for $2 \mathrm{~min}$, followed by multiple gradients to $90 \%$ at $20 \mathrm{~min}$; and the conditions were kept constant for $3 \mathrm{~min}$ and then changed to the initial conditions $(10 \% \mathrm{~B})$ after $5 \mathrm{~min}$, followed by a 7 -min isocratic wash at $10 \% \mathrm{~B}$ to re-equilibrate the column. The total chromatographic run time was $35 \mathrm{~min}$. The PDA detector scanning range was set from 220 to $400 \mathrm{~nm}$, and the chromatograms were processed at $325 \mathrm{~nm}$. A column temperature study was conducted using a longer LC program ( $45 \mathrm{mins}$ ) to enhance the separation of diCQA geometrical isomers. The column oven temperature was set at $30,35,40,45,50,55$, and $60^{\circ} \mathrm{C}$.

\section{Results and Discussion}

In this study, positional isomers of diCQAs (Scheme 1) were UV irradiated, and the resulting samples were analyzed under reverse-phase chromatographic conditions using nine different column chemistries. Here, for each column, either methanol (containing $0.1 \%$ formic acid) or acetonitrile (containing $0.1 \%$ formic acid) was used as the mobile phase/eluent $\mathrm{B}$, with the initial column oven temperature set at 30 and then $50^{\circ} \mathrm{C}$. Following analyses of the results, the use of aqueous methanol as the mobile phase/eluent B showed enhanced separation and, as such, the results discussed herein will be those obtained with methanol as part of the mobile phase composition [12]. The enhanced chromatographic separation using methanol as part of the binary solvent mixture is due to the weak eluent nature of methanol, which enhances the chromatographic separation of aromatic compounds by promoting longer retention within the column $[12,20]$. However, the results obtained with aqueous acetonitrile will also be referred to when necessary. In addition, unless stated otherwise, the results discussed herein are those obtained with the column oven temperature set at $30^{\circ} \mathrm{C}$.

The chromatograms of the nonirradiated $(0 \mathrm{~h})$ and irradiated $(4 \mathrm{~h})$ samples were compared and the retention times $\left(t_{R}\right)$ of the peak observed from the nonirradiated samples were used to identify the trans-isomers from each irradiated diCQA sample, for each column used to conduct the study (Figure 1). A summary of the chromatographic results is represented in Table 1, where the void volume/"dead time" was assessed for each column by the inspection of the chromatograms, and the resulting capacity factors $(k)$ for the trans- and cis-isomers are shown.

3.1. The Effect of Column Chemistry on the Separation of diCQA Geometrical Isomers. The results show that the chromatographic profile for the UV-irradiated sample of 1,3diCQA is consistent on both the bi-phenyl and $\mathrm{C}_{18}$ column matrices (Figure 2(a)) and consistent with the results achieved by Clifford et al. [4]. On both the bi-phenyl and $\mathrm{C}_{18}$ column matrices (Figure 2(a)), two mono-cis-isomers $\left(\mathbf{M}^{*}\right.$ and $\mathbf{M}^{*}$ ) were observed to elute after their respective trans-counterpart (T). According to Clifford et al., a peak of minor intensity is 

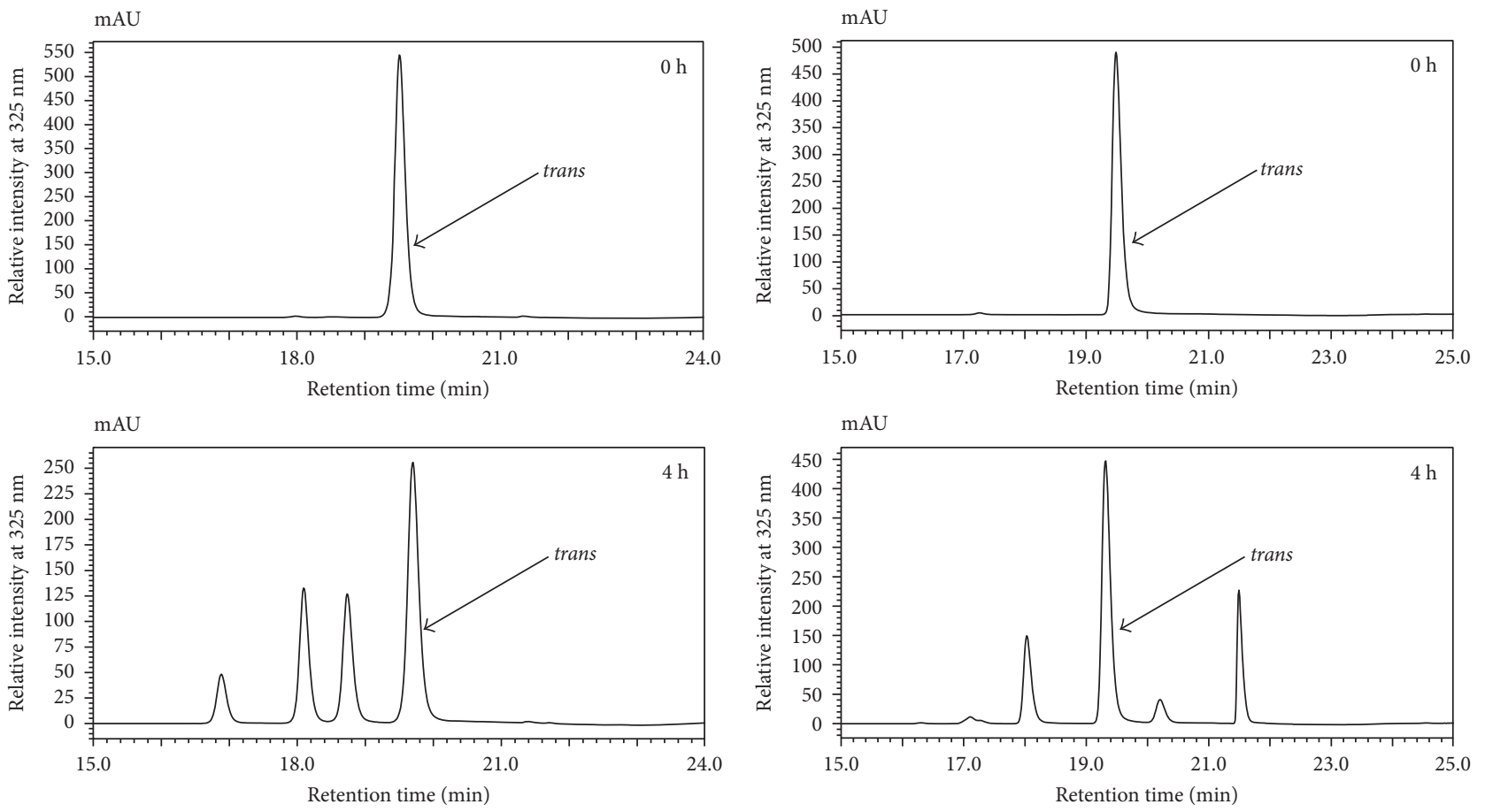

(a)

(b)

FIGURE 1: Comparisons of HPLC-PDA chromatogram profiles of nonirradiated ( $0 \mathrm{~h}$ ) and UV irradiated (4h) samples of (a) 3,5-diCQA analyzed on a Pinnacle $\mathrm{C}_{18}$ column and (b) 4,5-diCQA analyzed on a Viva $\mathrm{C}_{18}$ column, using aqueous methanol as the mobile phase composition.

considered the di-cis-isomer [4]; thus, from Figure 2(a), C was annotated as the di-cis-isomer. In a study by Zheng et al., where the 3,5-diCQA geometrical isomers were separated by ion mobility, the photoisomerization study revealed that the di-cis-isomer forms directly from both the mono-cis-isomers [15]. In this study, the di-cis-isomer was retained longer on both column matrices (bi-phenyl and $\mathrm{C}_{18}$ ), suggesting the resolution of the di-cis-isomer from the other isomers (Figure 2(a)). Thus, for simplicity, the elution order of the various isomers for 1,3-diCQA is referred to as $\mathbf{T} \mathbf{M}^{*} \mathbf{M}^{\#} \mathbf{C}$, where for all diCQAs, $\mathbf{T}$ represents the di-trans, $\mathbf{M}^{*}$ represents the first eluting mono-cis-isomer, $\mathbf{M}^{\#}$ represents the second eluting mono-cis-isomer, and $\mathbf{C}$ represents the di-cis-isomer. Furthermore, the elution profile/order for all diCQAs is summarized in Table 2.

For the UV-irradiated sample of 1,5-diCQA, four peaks (corresponding to the four isomers) were observed when using the bi-phenyl and $\mathrm{C}_{18}$ column matrices, and it is apparent that the elution profile/order differs between the two column matrices (Figure 2(b)). On the $\mathrm{C}_{18}$ column matrix, two peaks, the first mono-cis-isomer $\left(\mathbf{M}^{*}\right)$ and the di-cis-isomer $(\mathbf{C})$, elute before the trans-isomer $(\mathbf{T})$ and the fourth peak, the second mono-cis-isomer $\left(\mathbf{M}^{\#}\right)$, elutes after the di-trans-isomer, resulting in the elution order $\mathbf{M}^{*} \mathbf{C} \mathbf{T} \mathbf{M}^{\#}$ (Table 2). However, on the bi-phenyl column, the cis-isomers are seen to elute after the trans-isomer, resulting with the elution order $\mathbf{T} \mathbf{M}^{*} \mathbf{C M}^{\#}$ (Figure 2(b)) (Table 2). The elution order, $\mathbf{T} \mathbf{M}^{*} \mathbf{C M}^{\#}$, observed on the bi-phenyl columns was similar to the elution order observed by
Clifford et al., using a phenyl-hexyl column matrix [4]. The consistency observed on the phenyl-containing column matrices (bi-phenyl versus phenyl-hexyl) suggests the possible role of $\pi-\pi$ interactions in the separation of these aromatic isomers $[12,16,17,21]$. In contrast, differences in the elution order amongst the $\mathrm{C}_{18}$ columns were observed (Figure 3). Instead of the elution order $\mathbf{M}^{*} \mathbf{C T} \mathbf{M}^{\#}$ seen in Figure 2(b) using a Raptor $\mathrm{C}_{18}$, the Ultra $\mathrm{C}_{18}$ column produced the elution order $\mathbf{C M}^{*} \mathbf{T} \mathbf{M}^{\#}$ (Figure 3 ).

The elution order observed for the UV-irradiated standard of 3,4-diCQA demonstrated a relatively identical elution profile, on columns showing enhanced separation of the four isomers. Where the first monocis-isomer $\left(\mathbf{M}^{*}\right)$ elutes before the trans-isomer $(\mathbf{T})$, the trans-isomer is followed by the second mono-cis-isomer $\left(\mathbf{M}^{\#}\right)$ and lastly followed by the di-cis-isomer $(\mathbf{C})$, resulting in the elution order $\mathbf{M}^{*} \mathbf{T} \mathbf{M}^{\#} \mathbf{C}$ (Figure 2(c)) (Table 2). The similar elution order observed on both the bi-phenyl and $\mathrm{C}_{18}$ column matrices can be attributed to the stereochemistry at positions 3 and 4 on the quinic acid unit (Scheme 1, Figure 2(c)). The similarities in the spatial arrangement of the caffeic acid units at these positions on the quinic acid unit could possibly result in comparable interactions with their surrounding environments (i.e., mobile and stationary phase). In addition, the similar elution profile seen on both the bi-phenyl and $\mathrm{C}_{18}$ column matrices for the UV-irradiated sample of 1,3-diCQA $\left(\mathbf{T} \mathbf{M}^{*} \mathbf{M}^{\#} \mathbf{C}\right.$ ) (Figure 2(a), Table 2) can also be attributed to the identical spatial arrangement at positions 1 and 3 on 


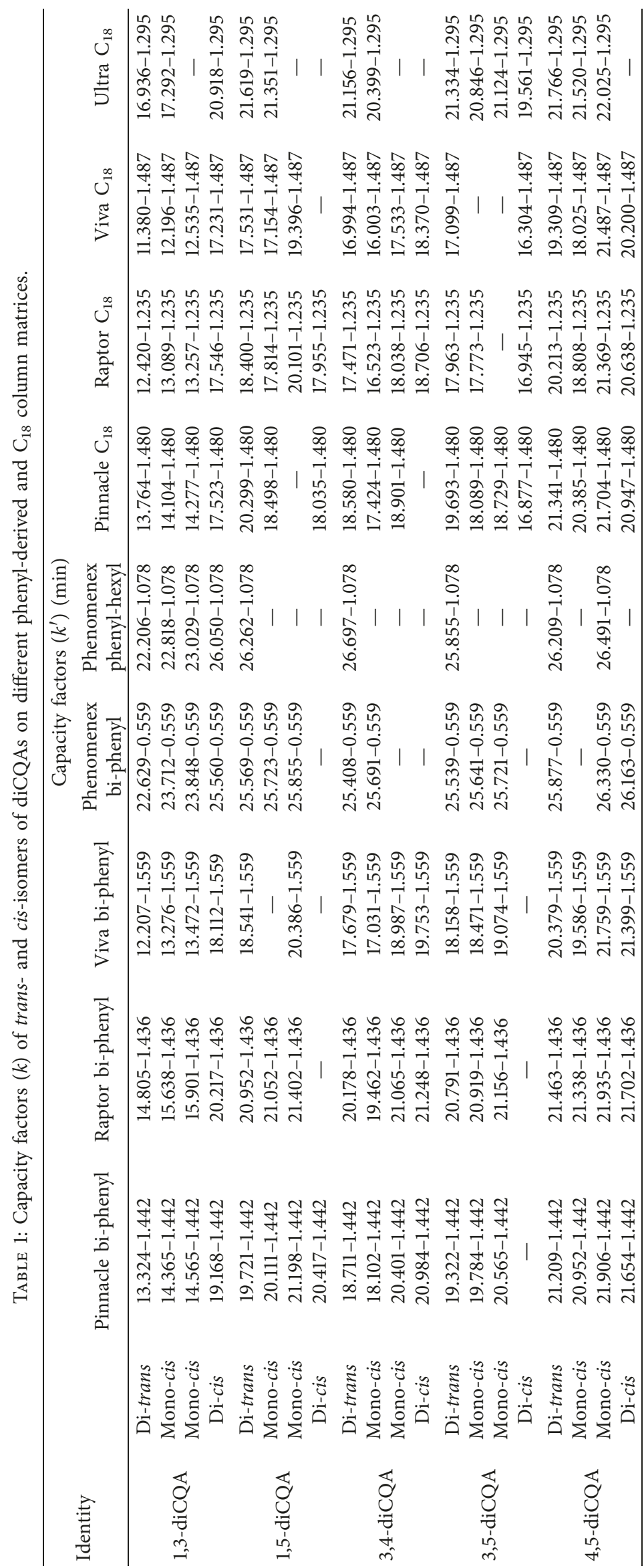



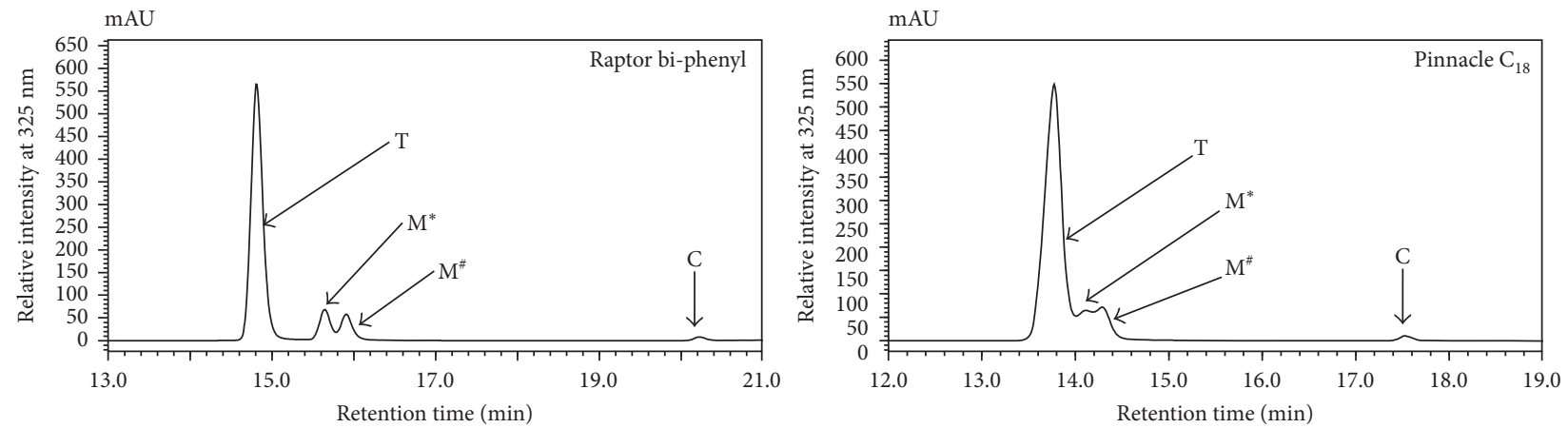

(a)
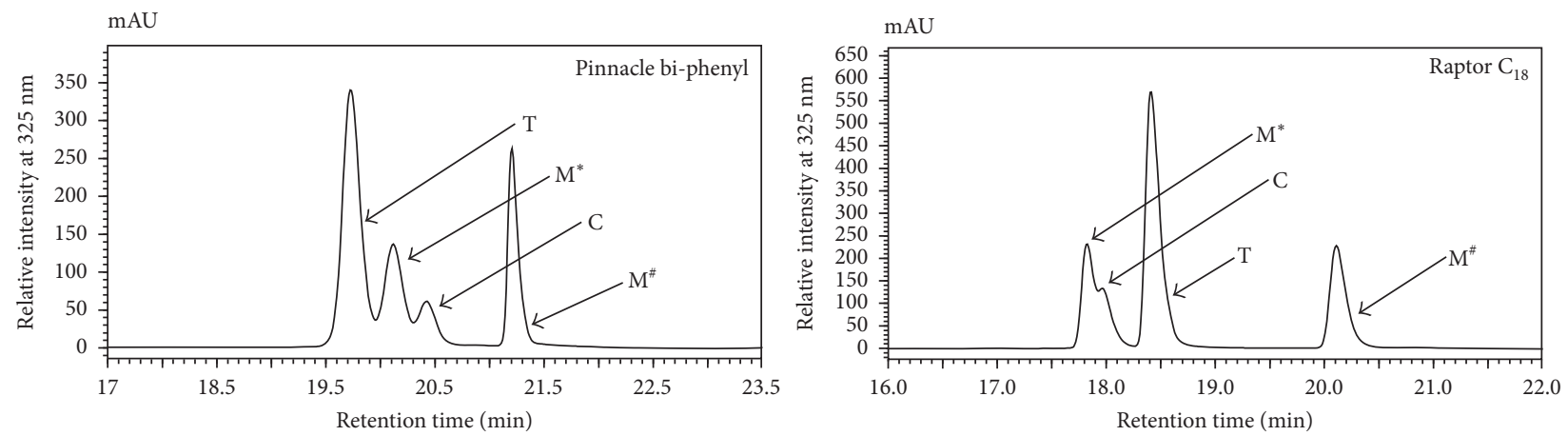

(b)
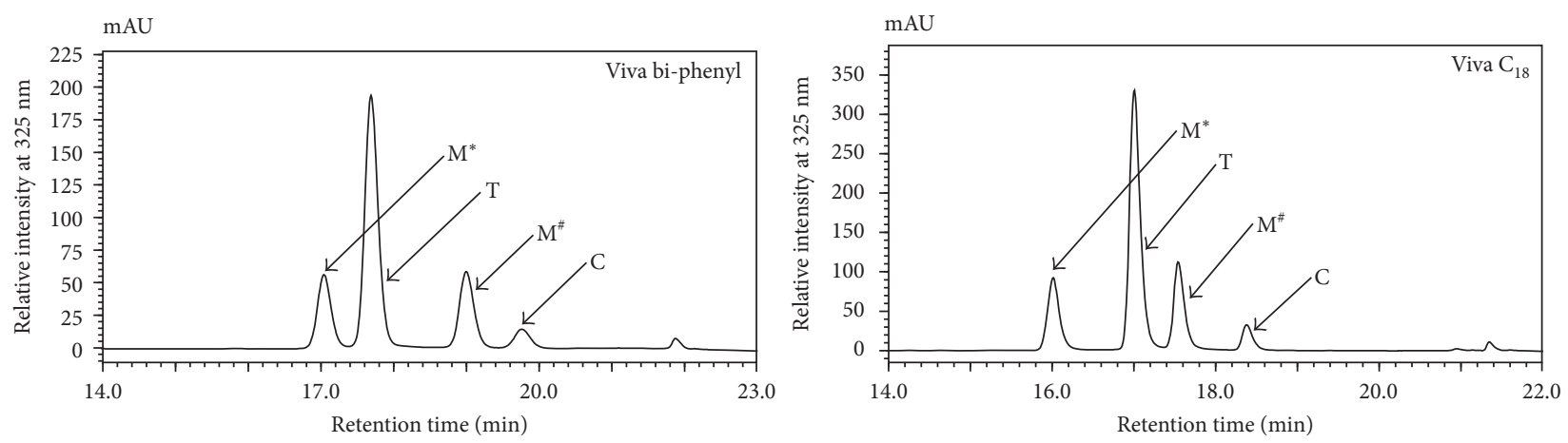

(c)
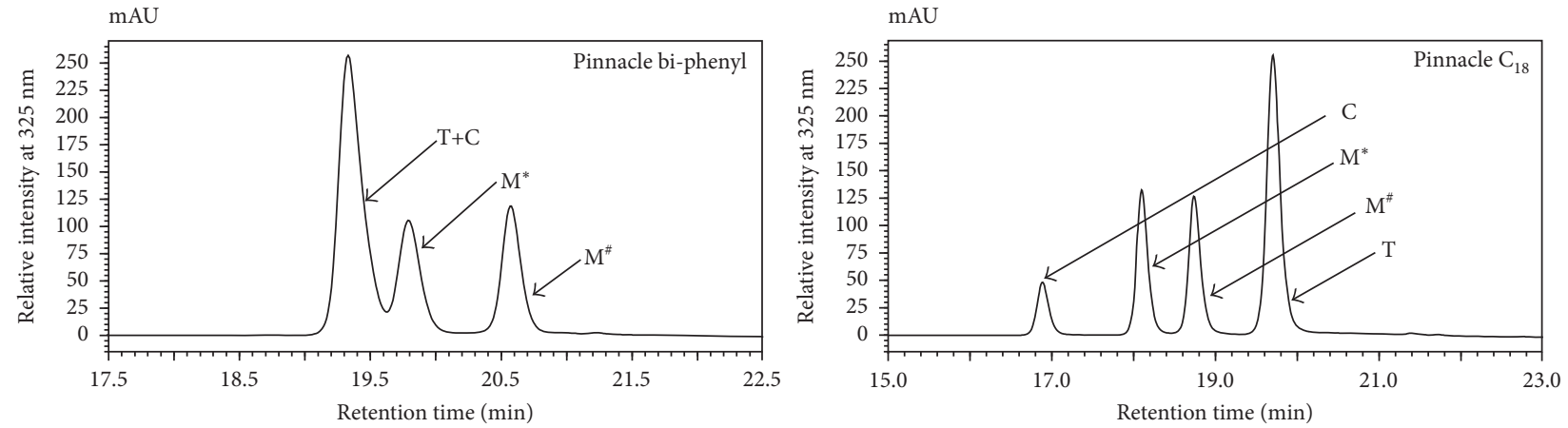

(d)

FIgURE 2: Continued. 

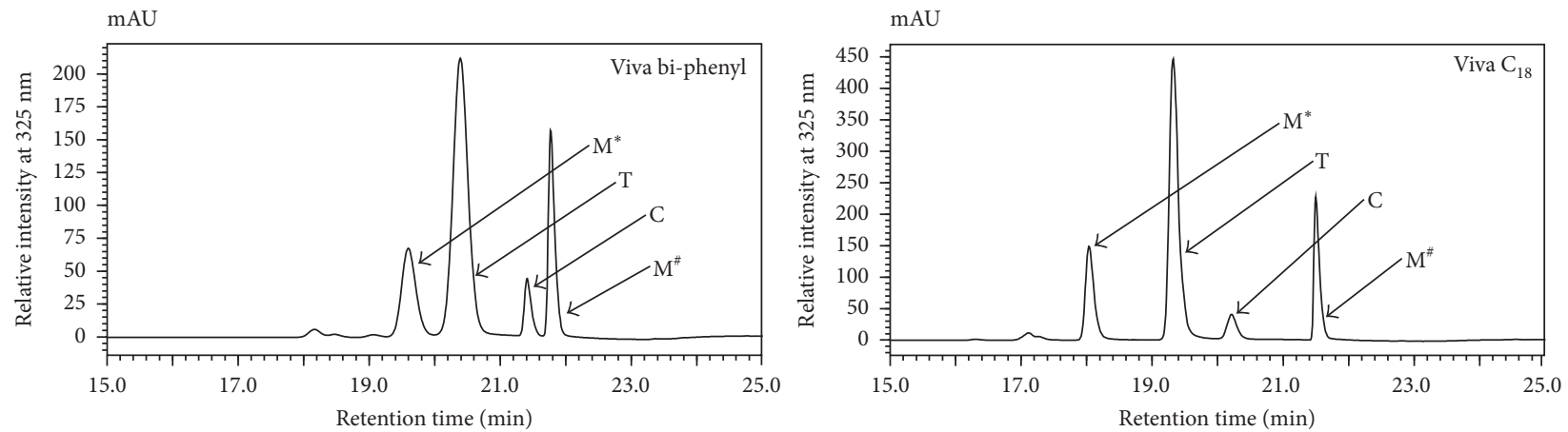

(e)

FIGURE 2: Overlaid HPLC-PDA chromatograms demonstrating the chromatographic separation of UV-irradiated samples of (a) 1,3-diCQA, (b) 1,5-diCQA, (c) 3,4-diCQA, (d) 3,5-diCQA, and (e) 4,5-diCQA analyzed on either a bi-phenyl or $\mathrm{C}_{18}$ column matrix. The figure shows differences and similarities in the elution profiles, dependent on either the bi-phenyl or $\mathrm{C}_{18}$ column matrix. Trepresents the di-trans-isomer, $\mathbf{M}^{*}$ represents the first eluting mono-cis-isomer, $\mathbf{M}^{\#}$ represents the second eluting mono-cis-isomer, and $\mathbf{C}$ represents the di-cis-isomer.

TABLE 2: Elution order of the trans- and cis-isomers of diCQAs on different column matrices (phenyl versus alkyl) using aqueous methanol as the eluent post optimization.

\begin{tabular}{|c|c|c|c|c|c|c|c|c|c|c|}
\hline & \multicolumn{9}{|c|}{ Column chemistry } \\
\hline & & $\begin{array}{l}\text { Pinnacle } \\
\text { bi-phenyl }\end{array}$ & $\begin{array}{c}\text { Raptor } \\
\text { bi-phenyl }\end{array}$ & $\begin{array}{c}\text { Viva } \\
\text { bi-phenyl }\end{array}$ & $\begin{array}{c}\text { Phenomenex } \\
\text { bi-phenyl }\end{array}$ & $\begin{array}{l}\text { Phenomenex } \\
\text { phenyl-hexyl }\end{array}$ & $\begin{array}{c}\text { Pinnacle } \\
\mathrm{C}_{18}\end{array}$ & $\begin{array}{c}\text { Raptor } \\
\mathrm{C}_{18}\end{array}$ & $\begin{array}{l}\text { Viva } \\
\mathrm{C}_{18}\end{array}$ & $\begin{array}{l}\text { Ultra } \\
\mathrm{C}_{18}\end{array}$ \\
\hline \multirow{5}{*}{$\begin{array}{l}\text { Elution } \\
\text { order of } \\
\text { isomers }\end{array}$} & 1,3-diCQA & $\mathbf{T M}^{*} \mathbf{M}^{\#} \mathbf{C}$ & $\mathbf{T M}^{*} \mathbf{M}^{\#} \mathbf{C}$ & $\mathbf{T M}^{*} \mathbf{M}^{\#} \mathbf{C}$ & $\mathbf{T M}^{*} \mathbf{M}^{\#} \mathbf{C}$ & $\mathbf{T M}^{*} \mathbf{M}^{\#} \mathbf{C}$ & $\mathbf{T M}^{*} \mathbf{M}^{\#} \mathbf{C}$ & $\mathbf{T M}^{*} \mathbf{M}^{\#} \mathbf{C}$ & $\mathbf{T M}^{*} \mathbf{M}^{\#} \mathbf{C}$ & $\mathbf{T M}^{*} \mathbf{M}^{\#} \mathbf{C}$ \\
\hline & 1,5-diCQA & $\mathrm{TM}^{*} \mathbf{C M}^{\#}$ & $\mathbf{T M}^{*} \mathbf{C M}^{\#}$ & $\mathbf{T M}^{*} \mathbf{C M}^{\#}$ & $\mathbf{T} \mathbf{M}^{*} \mathbf{M}^{\#}$ & TM$^{*}$ & $\mathbf{C M}^{*} \mathbf{T}$ & $\mathbf{M}^{*} \mathbf{C T M}^{\#}$ & $\mathbf{M}^{*} \mathbf{T} \mathbf{M}^{\#}$ & $\mathbf{C M}^{*} \mathbf{T M}^{\#}$ \\
\hline & 3,4-diCQA & $\mathbf{M}^{*} \mathbf{T} \mathbf{M}^{\#} \mathbf{C}$ & $\mathbf{M}^{*} \mathbf{T} \mathbf{M}^{\#} \mathbf{C}$ & $\mathbf{M}^{*} \mathbf{T} \mathbf{M}^{\#} \mathbf{C}$ & TM* C & $\mathbf{M}^{*} \mathbf{T} \mathbf{M}^{\#}$ & $\mathbf{M}^{*} \mathbf{T C M}^{\#}$ & $\mathbf{M}^{*} \mathbf{T} \mathbf{M}^{\#} \mathbf{C}$ & $\mathbf{M}^{*} \mathbf{T} \mathbf{M}^{\#} \mathbf{C}$ & $\mathbf{M}^{*} \mathbf{T} \mathbf{M}^{\#}$ \\
\hline & 3,5-diCQA & $\mathbf{T C M}^{*} \mathbf{M}^{\#}$ & $\mathbf{T C M}^{*} \mathbf{M}^{\#}$ & $\mathbf{T C M}^{*} \mathbf{M}^{\#}$ & $\mathbf{T M}^{*} \mathbf{M}^{\#}$ & $\mathrm{~T}$ & $\mathbf{C M}^{*} \mathbf{M}^{\#} \mathbf{T}$ & $\mathbf{C M}^{*} \mathbf{T}$ & $\mathrm{CTM}^{\#}$ & $\mathbf{C M}^{*} \mathbf{M}^{\#} \mathbf{T}$ \\
\hline & 4,5-diCQA & $\mathbf{M}^{*} \mathbf{T C M}^{*}$ & $\mathbf{M}^{*} \mathbf{T C M}^{\#}$ & $\mathbf{M}^{*} \mathbf{T C M}^{\#}$ & $\mathbf{T M}^{*} \mathbf{C M}^{\#}$ & $\mathbf{M}^{*} \mathbf{T} \mathbf{M}^{\#}$ & $\mathbf{M}^{*} \mathbf{C T M}^{\#}$ & $\mathbf{M}^{*} \mathbf{T C M}^{\#}$ & $\mathbf{M}^{*} \mathbf{T C M}^{\#}$ & $\mathbf{M}^{*} \mathbf{C T M}^{\#}$ \\
\hline
\end{tabular}

Trepresents the di-trans-isomer; $\mathbf{M}^{*}$ represents the first eluting mono-cis-isomer; $\mathbf{M}^{\#}$ represents the second eluting mono-cis-isomer; $\mathbf{C}$ represents the di-cisisomer.
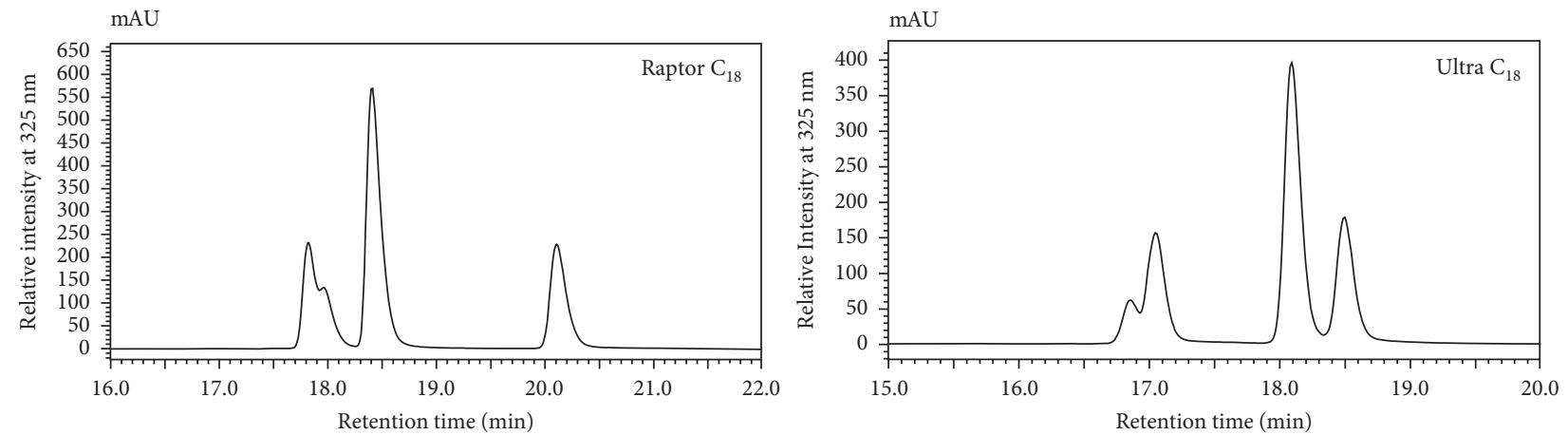

FIGURE 3: HPLC-PDA chromatograms of the UV-irradiated sample of 1,5-diCQA, showing different elution profiles on $\mathrm{C}_{18}$ column matrices. The Raptor $\mathrm{C}_{18}$ shows the elution profile $\mathbf{M}^{*} \mathbf{C} \mathbf{T} \mathbf{M}^{\#}$, and the Ultra $\mathrm{C}_{18}$ shows the elution profile $\mathbf{C M}^{*} \mathbf{T} \mathbf{M}^{\#}$.

the quinic acid unit to which the caffeic acid units are esterified. Furthermore, in their study, Clifford et al. only observed three geometrical isomers (instead of four) for the UV-irradiated sample of 3,4-diCQA using a phenyl-hexyl column [4]. Here, the elution order observed was $\mathbf{M}^{*} \mathbf{T} \mathbf{M}^{\#}$ instead of the elution order $\mathbf{M}^{*} \mathbf{T} \mathbf{M}^{\#} \mathbf{C}$ observed in our study and discussed above. Results observed by Clifford et al. were also observed in our study when the phenyl-hexyl column was used with aqueous acetonitrile as the eluent (see Supplementary Figure S1), thus suggesting the possible coelution of the di-cis-isomer under these conditions.

When the UV-generated geometrical isomers of 3,5diCQA were analyzed on the phenyl-containing column matrices (at a column temperature of $30^{\circ} \mathrm{C}$ ), only three peaks (instead of four peaks) were observed and resulted with the elution order $\mathbf{T} \mathbf{M}^{*} \mathbf{M}^{\#}$ (Figure 2(d), Table 2), as observed by Clifford et al. [4]. On the $\mathrm{C}_{18}$ column matrices, the elution order $\mathbf{C M}^{*} \mathbf{M}^{\#} \mathbf{T}$ was observed (Figure 2(d), Table 2). 

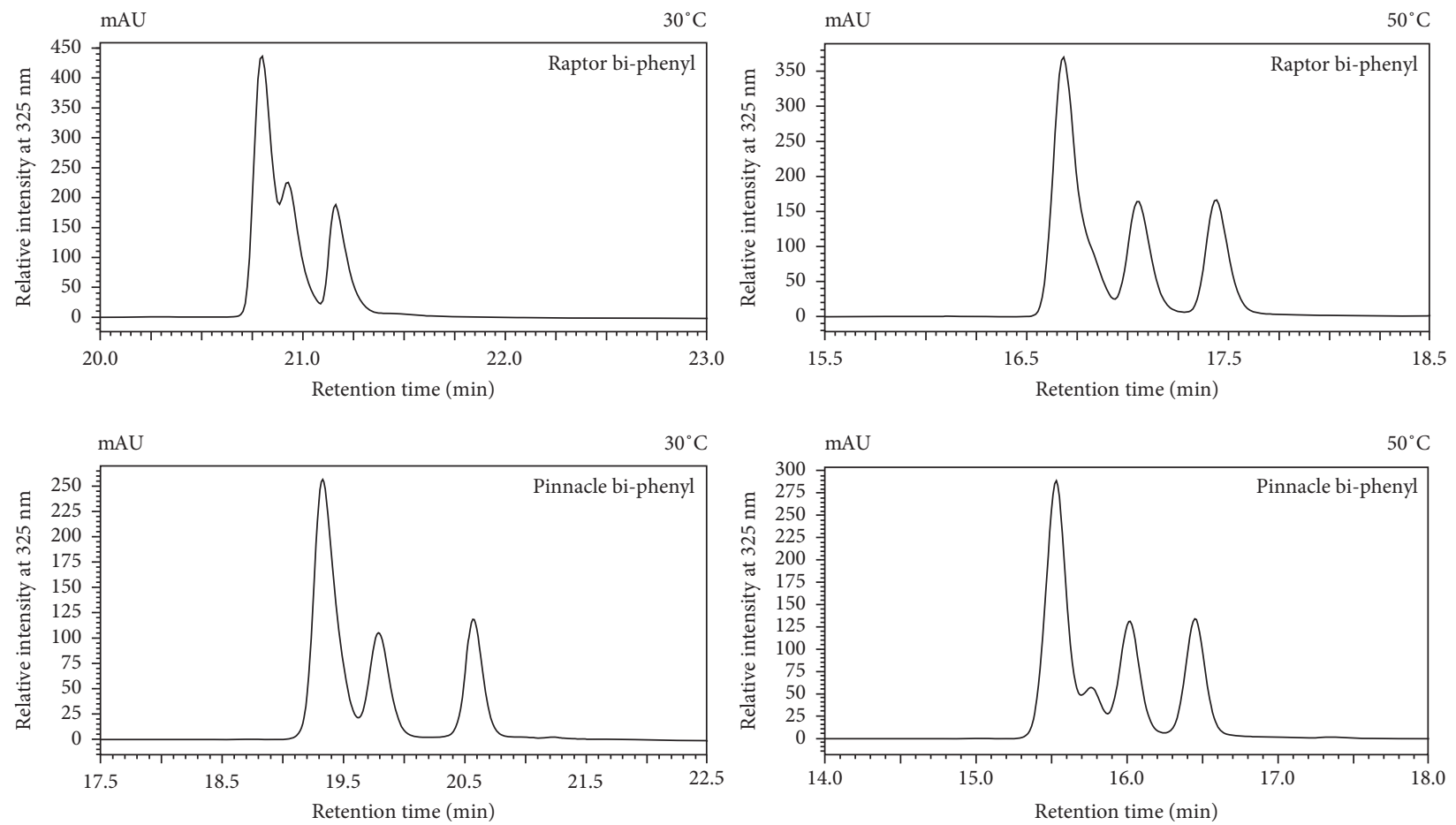

FIgURE 4: Comparison of HPLC-PDA chromatograms of UV-irradiated sample of 3,5-diCQA achieved with bi-phenyl columns, showing the enhanced separation of isomers due to an increase in column temperature.

Although not commonly considered a key parameter in reverse-phase chromatography, high column temperatures have been shown to enhance the separation due to a decrease in the viscosity of the mobile phase. In addition, retention factors are dependent on the distribution coefficients $\left(k_{d}\right)$ of the analytes, which are temperature dependent. An increase in temperature enhances the separation $[19,22,23]$. Thus, to achieve the separation of 3,5-diCQA geometrical isomers on the phenyl-containing column matrices, a column temperature of $50^{\circ} \mathrm{C}$ was introduced and resulted in the elution order $\mathbf{T C M} \mathbf{M}^{*} \mathbf{M}^{\#}$ (Figure 4) instead of the elution order $\mathbf{T} \mathbf{M}^{*} \mathbf{M}^{\#}$ seen in Figure 2(d). The consistency in the elution profile observed on the phenyl column matrices $\left(\mathbf{T} \mathbf{M}^{*} \mathbf{M}^{\#}\right)$ can be attributed to $\pi-\pi$ interactions which enhance separation in phenyl-containing columns $[12,16,17,20,21]$, and the elution profile differences observed between column matrices (phenyl versus alkyl matrices) can be attributed to the differences in spatial arrangements of the caffeoyl units at positions 3 and 5 on the quinic acid unit. For instance, the different spatial arrangements at these positions affect when cis-isomers elute on a phenyl column matrix (elutes after the trans-isomer; $\mathbf{T C M} \mathbf{M}^{*} \mathbf{M}^{\#}$ ) versus an alkyl column (elutes before the trans-isomer; $\left.\mathrm{CM}^{*} \mathbf{M}^{\#} \mathbf{T}\right)$.

Finally, the elution order for the geometrical isomers of 4,5-diCQA was seen to be $\mathbf{M}^{*} \mathbf{T} \mathbf{C M}^{\#}$, only on three bi-phenyl columns and two $\mathrm{C}_{18}$ columns as summarized in Table 2 and demonstrated in Figure 2(e). Interestingly, the other two $\mathrm{C}_{18}$ columns showed a different elution order (Figure 5); thus, instead of $\mathbf{M}^{*} \mathbf{T} \mathbf{C} \mathbf{M}^{\#}$ (Figure 5(a)), $\mathbf{M}^{*} \mathbf{C} \mathbf{T} \mathbf{M}^{\#}$ was observed
(Figure 5(b)). Furthermore, for this sample, Clifford et al. observed the elution order $\mathbf{T} \mathbf{M}^{*} \mathbf{C M}^{\#}$ when using a phenylhexyl column [4]. In our study, these results were also observed using a Phenomenex bi-phenyl column with acetonitrile as part of the mobile phase composition (Supplementary data, Figure S2). Thus, care must be taken when analyzing geometrical isomers of 4,5-diCQA on different column matrices.

Despite the inconsistent chromatographic elution profiles observed for the UV-irradiated sample of 4,5diCQA, what seems to be consistent is the later elution of the second eluting mono-cis-isomer $\left(\mathbf{M}^{\#}\right)$ (Figure 5). This is also evident in samples of 1,5-diCQA (Figure 2(b)) and 3,5-diCQA (Figure 2(d), Figure 4), especially when analyzed on phenyl-containing column matrices. This mono-cis-isomer could possibly be a cis-isomer at position 5 on the quinic acid unit. According to Clifford et al., a cisgeometry at position 5 on the quinic acid for mono-acyl chlorogenic acids results in intramolecular hydrogen bonding at two positions; (1) the caffeoyl carbonyl $(\mathrm{C}=\mathrm{O})$ group and the 4-OH group on the quinic acid unit and (2) the caffeoyl $3^{\prime}-\mathrm{OH}$ group and the carbonyl group at position 1 on the quinic acid unit [4], thus rendering the molecules less hydrophilic due to unavailable hydroxyl groups and the compact nature of the molecule. In this study, it is uncertain to what extent the above applies to the diCQAs (Supplementary data, Figure S3).

3.2. The Effect of Column Temperature on the Separation of diCQA Geometrical Isomers. From the above results, it is 

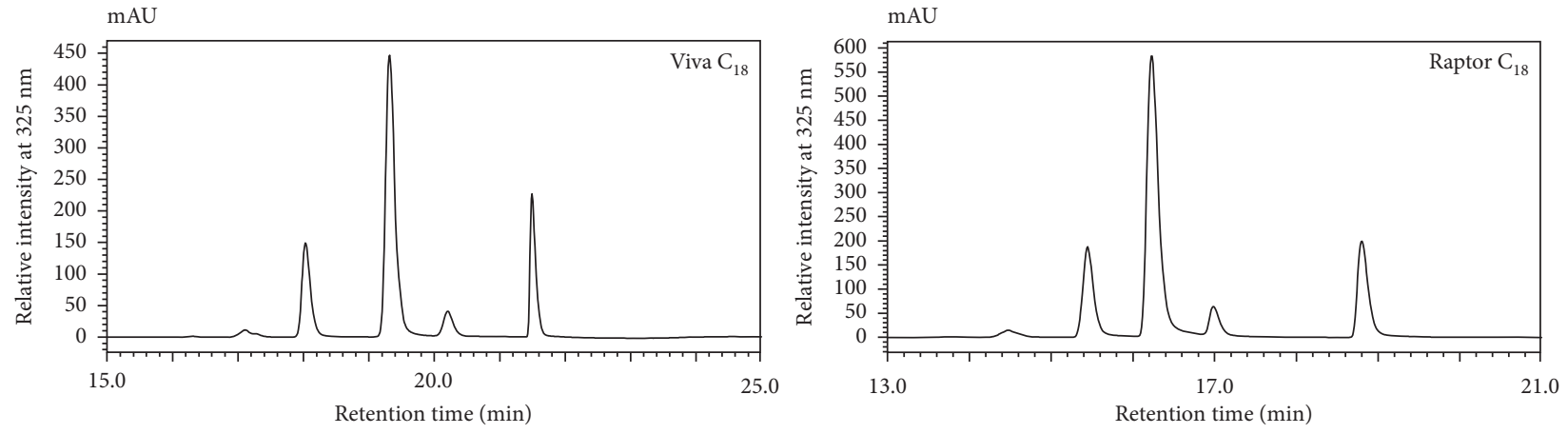

(a)
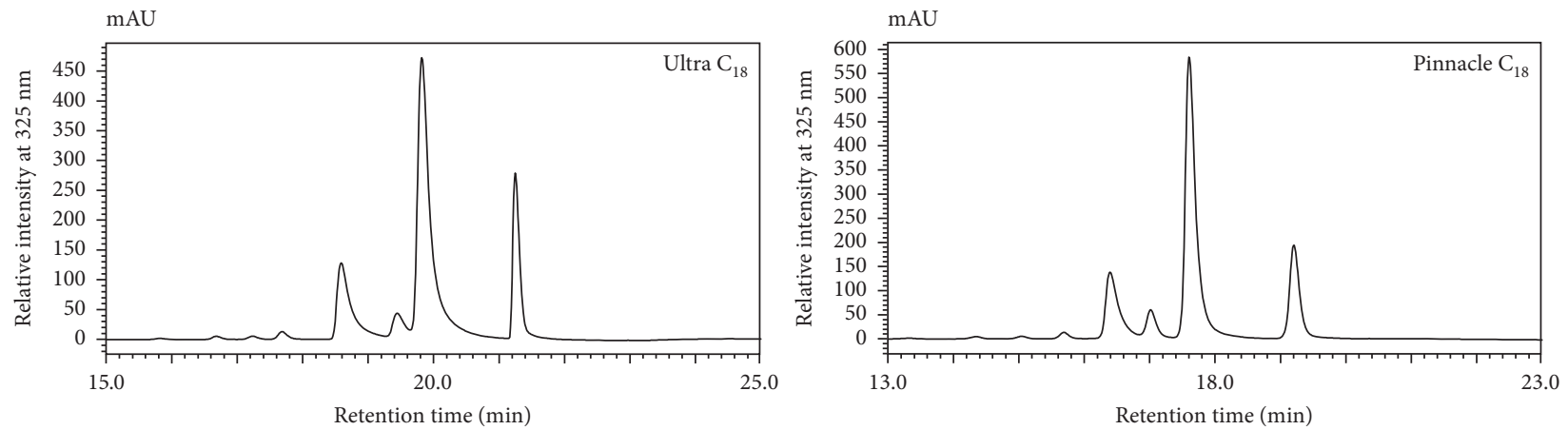

(b)

FIgURE 5: HPLC-PDA chromatograms of UV-irradiated sample of 4,5-diCQA showing different elution profiles, (a) $\mathbf{M} * \mathbf{T C M}{ }^{\#}$ and (b) $\mathbf{M} * \mathbf{C T M}^{\#}$, on four $\mathrm{C}_{18}$ column matrices.

apparent that determining the elution order of diCQA geometrical isomers on different reverse-phase column matrices shows inconsistencies (Table 2). Within the $\mathrm{C}_{18}$ column matrices, different chromatographic elution profiles were observed, and the Ultra $\mathrm{C}_{18}$ column showed the worst performance when analysis was conducted with the column temperature set at $30^{\circ} \mathrm{C}$. As such, column temperature was varied $\left(30-60^{\circ} \mathrm{C}\right)$ to enhance the separation of the diCQA geometrical isomers on the Ultra $\mathrm{C}_{18}$ column (Figure 6). From Figure 6, an increase in column temperature showed a positive effect on the resolution of the geometrical isomers of 1,3-diCQA, 1,5-diCQA, 3,5diCQA, and 4,5-diCQA. An increase in temperature resulted in the earlier elution of analytes and resolution of the UV-irradiated diCQA geometrical isomers (Figure 6). Similar results were observed by Nguyen et al., whereby a pharmaceutical cocktail was chromatographically separated at temperatures $30^{\circ} \mathrm{C}$ and $90^{\circ} \mathrm{C}$, and the temperature at $90^{\circ} \mathrm{C}$ enhanced the separation and decreased the analysis time [19]. Furthermore, in our study the UV-irradiated sample of 3,4-diCQA showed the separation and resolution of only three isomers, suggesting coelution of the di-cis-isomer. For this sample (UV-irradiated sample of 3,4-diCQA), the elution order $\left(\mathbf{M}^{*} \mathbf{T} \mathbf{M}^{\#}\right)$ was also observed using the phenyl-hexyl column coupled with aqueous acetonitrile as part of the mobile phase (Supplementary data, Figure S1). The results obtained in this temperature study suggest that some column matrices are incapable of separating/distinguishing all the available isomers in the sample even post optimization of the chromatographic parameters.

\section{Conclusion}

This study demonstrates that positional isomers of diCQA samples produce three cis-isomers post UV irradiation, and separation of these isomers is dependent on optimization of primary chromatographic parameters. As such, column chemistry, mobile phase composition, and column temperature influence the chromatographic elution profile of the structurally related compounds, thus hindering identification. From the above results, it is apparent that determining the elution profile/order of diCQA geometrical isomers on different reverse-phase column matrices (phenyl versus alkyl) shows inconsistencies. However, a relatively consistent elution order was observed using the phenyl-containing column matrices, suggesting the important role of $\pi-\pi$ interactions in the separation of the respective diCQA geometrical isomers. The results show different elution profiles between $\mathrm{C}_{18}$ column matrices from different column suppliers, suggesting that column manufacturing is not standardized.

The study also shows that column temperature can be used to enhance the separation of the isomers. Furthermore, the number of observed isomers depends on the capability of the column to distinguish the isomers. For instance, using the Ultra $\mathrm{C}_{18}$ column, the separation of the 3,4-diCQA geometrical isomers was enhanced by the introduction of column temperature; however, the di- 

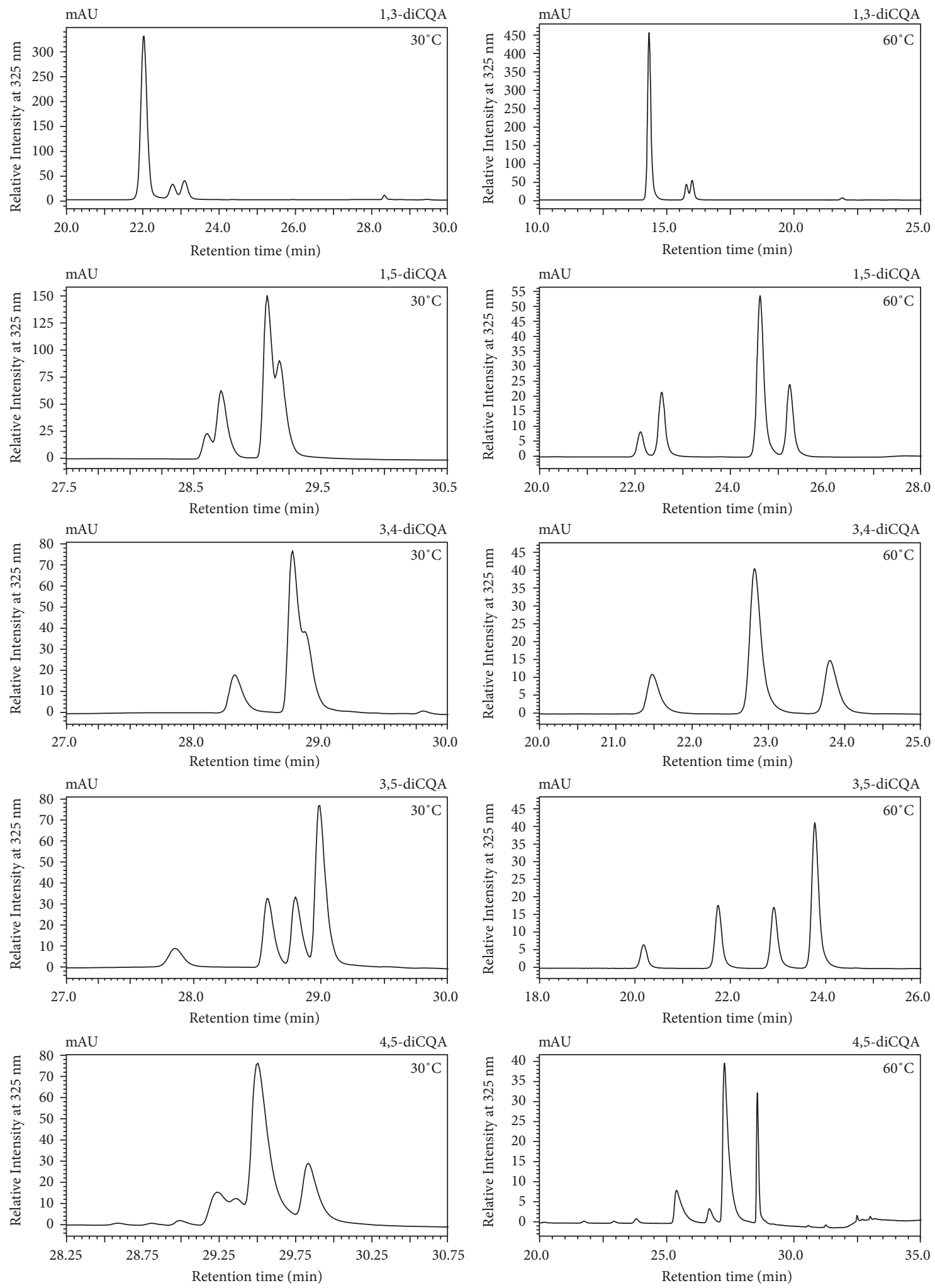

FIGURE 6: Graphical representation of the influence of temperature in enhancing the resolution of diCQA geometrical isomers as indicated. The figure shows that an increase in column temperature enhances the separation of diCQA geometrical isomers.

cis-isomer was not observed on the chromatogram, suggesting coelution of the di-cis-isomer. Lastly, the study highlights that chromatographic elution order/profile cannot be relied upon to fathom the complexity of isomeric plant metabolites and that more advanced analytical methods need to be developed to achieve this goal. Advancement in analytical approaches can include hyphenation of high-temperature liquid chromatography 
(HTLC) to metal binding-based MS differentiation [14] or hyphenation of HTLC to ion mobility MS [15].

\section{Conflicts of Interest}

The authors declare that there are no conflicts of interest.

\section{Supplementary Materials}

Figure S1: Elution profile of 3,4-diCQA geometrical isomers obtained using Phenomenex phenyl-hexyl and acetonitrile as the mobile phase composition. Figure S2: Elution profile of 4,5-diCQA geometrical isomers obtained using Phenomenex bi-phenyl and acetonitrile as the mobile phase composition. Figure S3: Energy optimized structure of geometrical isomers of 3,5 diCQA. The structures were optimized using the B3LYP/6-311+G(d,p) [11]. A) $3^{\text {trans }}, 5^{\text {trans }}$-diCQA (ditrans), B) $3^{\text {cis }}, 5^{\text {trans }}$-diCQA (mono-cis), C) $3^{\text {trans }}, 5^{\text {cis }}$-diCQA (mono-cis), and D) $3^{\text {cis }}, 5^{\text {cis }}$-diCQA (di-cis). (Supplementary Materials)

\section{References}

[1] M. N. Clifford, K. L. Johnston, S. Knight, and N. Kuhnert, "Hierarchical scheme for LC-MSn identification of chlorogenic acids," Journal of Agricultural and Food Chemistry, vol. 51, no. 10, pp. 2900-2911, 2003.

[2] H. M. Heyman, F. Senejoux, I. Seibert, T. Klimkait, V. J. Maharaj, and J. J. M. Meyer, "Identification of anti-HIV active dicaffeoylquinic- and tricaffeoylquinic acids in Helichrysum populifolium by NMR-based metabolomic guided fractionation," Fitoterapia, vol. 103, pp. 155-164, 2015.

[3] M. L. Salum and R. Erra-Balsells, "High purity cis-cinnamic acid preparation for studying physiological role of transcinnamic and cis-cinnamic acids in higher plants," Environmental Control in Biology, vol. 51, no. 1, pp. 1-10, 2013.

[4] M. N. Clifford, J. Kirkpatrick, N. Kuhnert, H. Roozendaal, and P. R. Salgado, "LC-MSn analysis of the cis isomers of chlorogenic acids," Food Chemistry, vol. 106, no. 1, pp. 379-385, 2008.

[5] R. Jaiswal, J. Kiprotich, and N. Kuhnert, "Determination of the hydroxycinnamate profile of 12 members of the Asteraceae family," Phytochemistry, vol. 72, no. 8, pp. 781-790, 2011.

[6] H. Karaköse, A. Müller, and N. Kuhnert, "Profiling and quantification of phenolics in Stevia rebaudiana leaves," Journal of Agricultural and Food Chemistry, vol. 63, no. 41, pp. 9188-9198, 2015.

[7] F. Parrino, A. Di Paola, V. Loddo, I. Pibiri, M. Bellardita, and L. Palmisano, "Photochemical and photocatalytic isomerization of trans-caffeic acid and cyclization of cis-caffeic acid to esculetin," Applied Catalysis B: Environmental, vol. 182, pp. 347-355, 2016.

[8] Y. Chen, S. Huang, F. Sun et al., "Transformation of cinnamic acid from trans-to cis-form raises a notable bactericidal and synergistic activity against multiple-drug resistant $M y$ cobacterium tuberculosis," European Journal of Pharmaceutical Sciences, vol. 43, no. 3, pp. 188-194, 2011.

[9] N. J. Farrer, J. A. Woods, L. Salassa et al., "A potent transDiimine platinum anticancer complex photoactivated by visible light," Angewandte Chemie International Edition, vol. 49, no. 47, pp. 8905-8908, 2010.

[10] E. F. Healy, J. Sanders, P. J. King, and W. E. Robinson, "A docking study of L-chicoric acid with HIV-1 integrase,"
Journal of Molecular Graphics and Modelling, vol. 27, no. 5, pp. 584-589, 2009.

[11] M. M. Makola, I. A. Dubery, G. Koorsen et al., "The effect of geometrical isomerism of 3,5-dicaffeoylquinic acid on its binding affinity to HIV-integrase enzyme: a molecular docking study," Evidence-Based Complementary and Alternative Medicine, vol. 2016, Article ID 4138263, 9 pages, 2016.

[12] K. Masike, F. Tugizimana, N. Ndlovu et al., "Deciphering the influence of column chemistry and mass spectrometry settings for the analyses of geometrical isomers of L-chicoric acid," Journal of Chromatography B, vol. 1052, pp. 73-81, 2017.

[13] W. S. Wong, D. Guo, X. L. Wang, Z. Q. Yin, B. Xia, and N. Li, "Study of cis-cinnamic acid in Arabidopsis thaliana," Plant Physiology and Biochemistry, vol. 43, no. 10-11, pp. 929-937, 2005.

[14] M. M. Makola, P. Steenkamp, I. A. Dubery, M. M. Kabanda, and N. E. Madala, "Preferential alkali metal adduct formation by cis geometrical isomers of dicaffeoylquinic acids allows for efficient discrimination from their trans isomers during ultrahigh-performance liquid chromatography/quadrupole timeof-flight mass spectrometry," Rapid Communications in Mass Spectrometry, vol. 30, no. 8, pp. 1011-1018, 2016.

[15] X. Zheng, R. S. Renslow, M. M. Makola et al., "Structural Elucidation of cis/trans dicaffeoylquinic acid photoisomerization using ion mobility spectrometry-mass spectrometry," Journal of Physical Chemistry Letters, vol. 8, no. 7, pp. 1381-1388, 2017.

[16] K. Croes, A. Steffens, D. Marchand, and L. Snyder, "Relevance of $\pi-\pi$ and dipole-dipole interactions for retention on cyano and phenyl columns in reversed-phase liquid chromatography," Journal of Chromatography A, vol. 1098, no. 1, pp. 123-130, 2005.

[17] P. G. Stevenson, K. J. Mayfield, A. Soliven et al., " $\pi$-Selective stationary phases:(I) influence of the spacer chain length of phenyl type phases on the aromatic and methylene selectivity of aromatic compounds in reversed phase high performance liquid chromatography," Journal of Chromatography A, vol. 1217 , no. 33 , pp. 5358-5364, 2010.

[18] T. J. Causon, K. Broeckhoven, E. F. Hilder, R. A. Shellie, G. Desmet, and S. Eeltink, "Kinetic performance optimisation for liquid chromatography: principles and practice," Journal of Separation Science, vol. 34, no. 8, pp. 877-887, 2011.

[19] D. T. Nguyen, D. Guillarme, S. Heinisch et al., "High throughput liquid chromatography with sub-2 $\mu \mathrm{m}$ particles at high pressure and high temperature," Journal of Chromatography A, vol. 1167, no. 1, pp. 76-84, 2007.

[20] J. Fibigr, D. Šatínský, and P. Solich, "A new approach to the rapid separation of isomeric compounds in a Silybum marianum extract using UHPLC core-shell column with F5 stationary phase," Journal of Pharmaceutical and Biomedical Analysis, vol. 134, pp. 203-213, 2017.

[21] P. Janas, S. Bocian, P. Jandera, T. Kowalkowski, and B. Buszewski, "Separation of flavonoids on different phenyl-bonded stationary phases-the influence of polar groups in stationary phase structure," Journal of Chromatography A, vol. 1429, no. 1, pp. 198-206, 2016.

[22] F. Lestremau, A. de Villiers, F. Lynen, A. Cooper, R. Szucs, and P. Sandra, "High efficiency liquid chromatography on conventional columns and instrumentation by using temperature as a variable: kinetic plots and experimental verification," Journal of Chromatography A, vol. 1138, no. 1-2, pp. 120-131, 2007.

[23] A. de Villiers, F. Lynen, and P. Sandra, "Effect of analyte properties on the kinetic performance of liquid chromatographic separations," Journal of Chromatography A, vol. 1216, no. 16, pp. 3431-3442, 2009. 

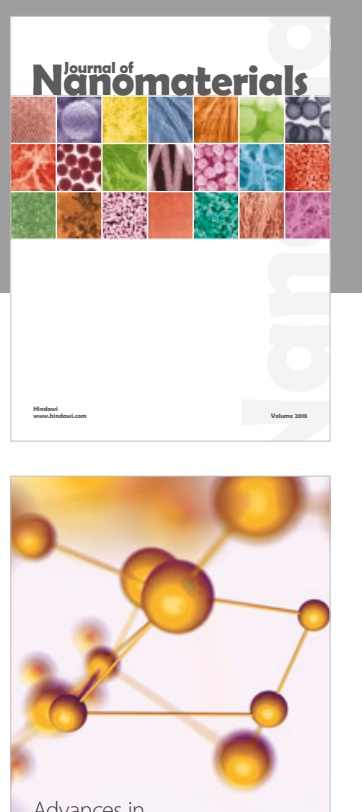

Physical Chemistry
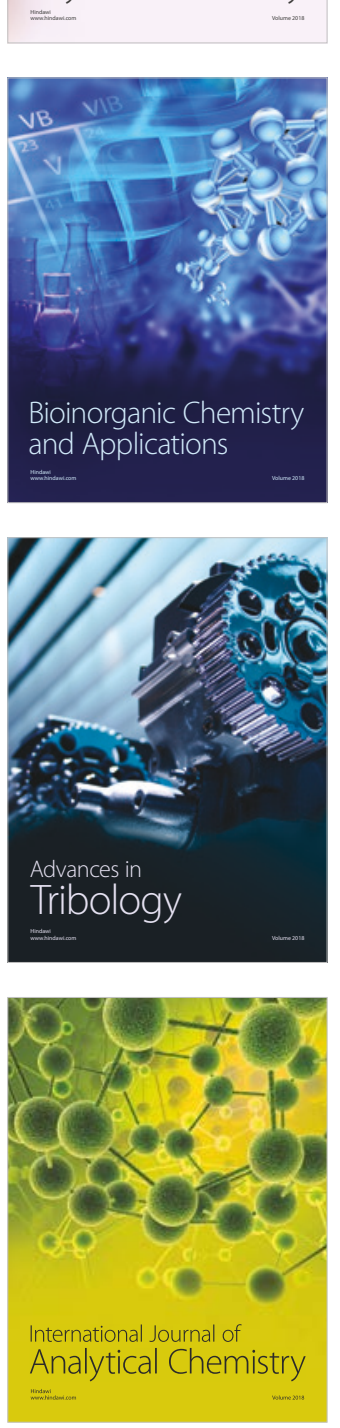

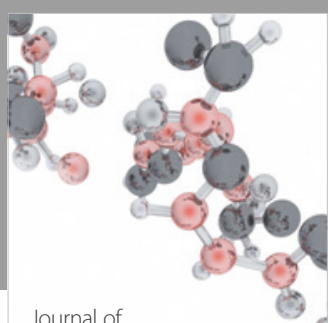

Analytical Methods

in Chemistry

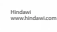

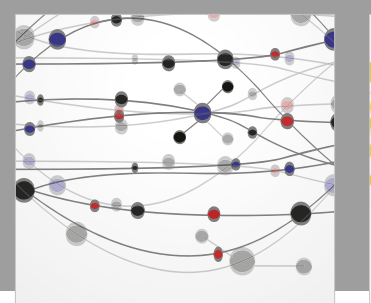

The Scientific World Journal

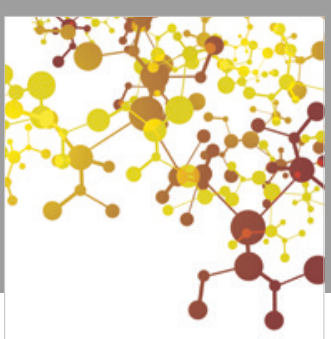

Journal of

Applied Chemistry
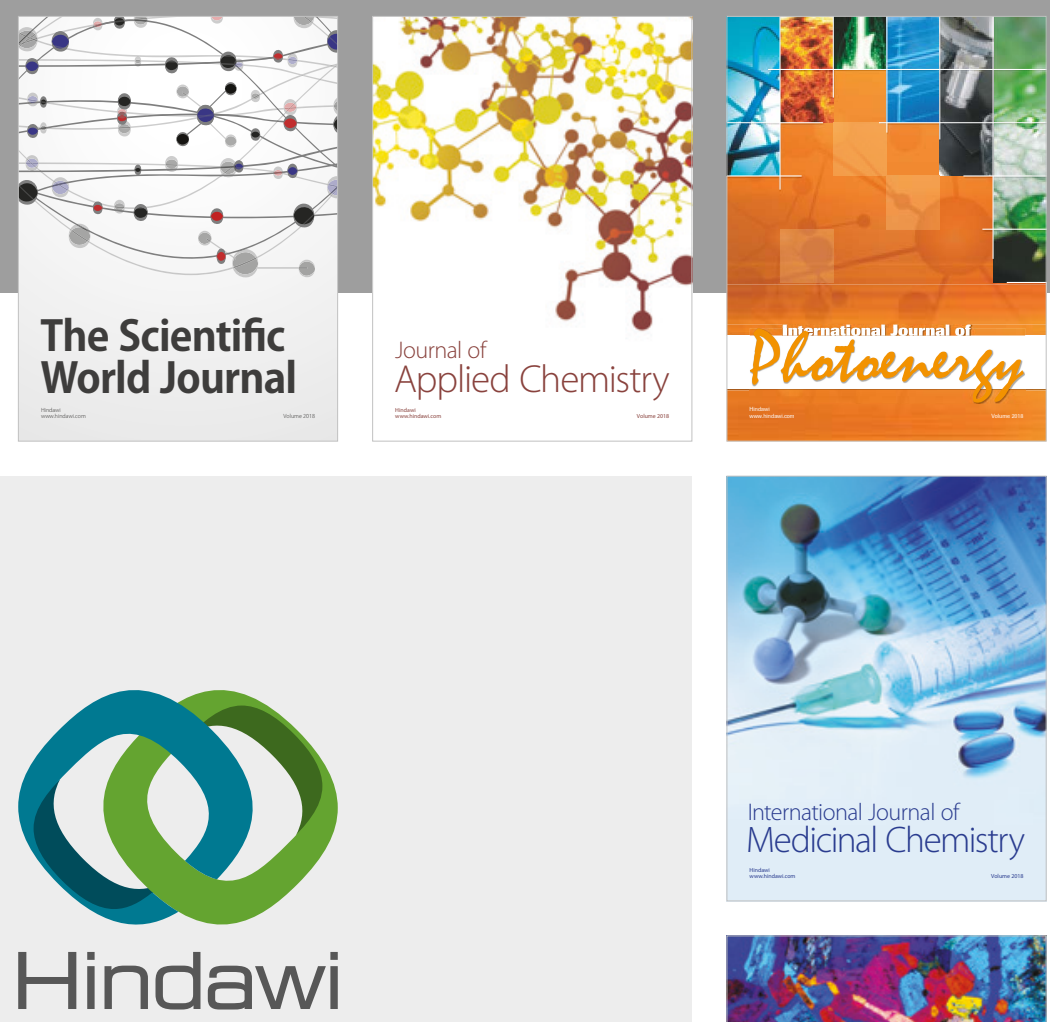

Submit your manuscripts at

www.hindawi.com
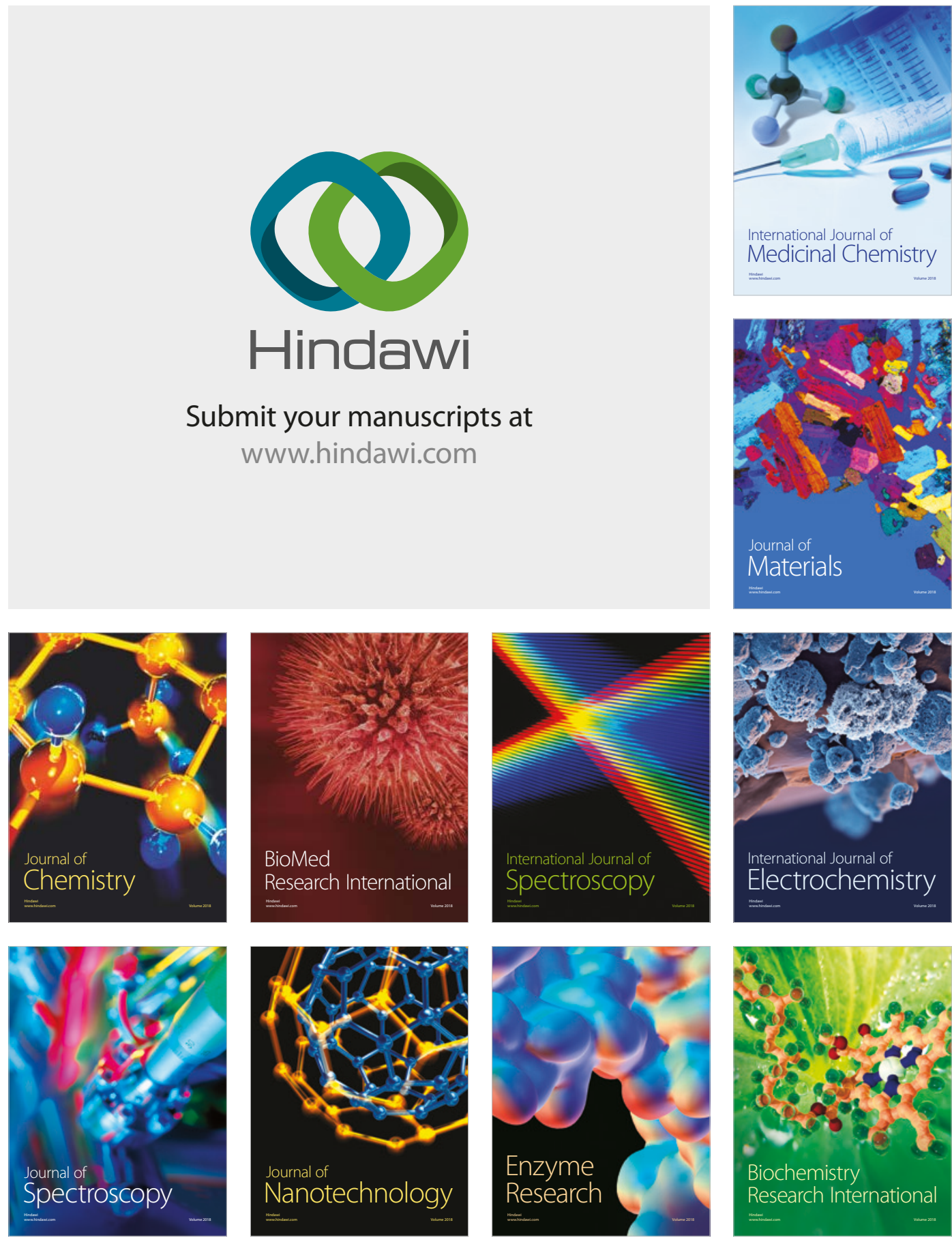
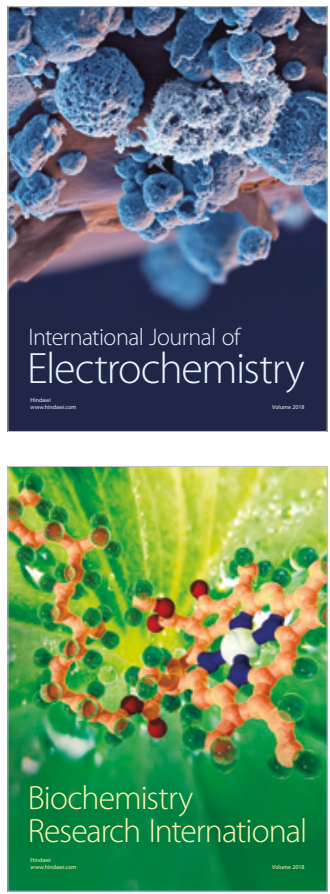